\title{
Accretion and wind dynamics in tidal disruption events
}

\author{
T. Mageshwaran ${ }^{1}$ and A. Mangalam ${ }^{2}$ \\ Indian Institute of Astrophysics, \\ Bangalore, India 560034, \\ email: ${ }^{1}$ mageshwaran@iiap.res.in, ${ }^{2}$ mangalam@iiap.res.in
}

\begin{abstract}
We have constructed self similar models of time dependent and non relativistic accretion disks in both sub and super-Eddington phase of TDEs with wind outflows for a general viscosity prescription which is a function of surface density of the disk $\Sigma_{d}$ and radius $r$. The physical parameters are black hole (BH) mass $M_{\bullet}$, specific orbital energy $E$ and angular momentum $J$, star mass $M_{\star}$ and radius $R_{\star}$. We have considered an accretion disk where matter is lost due to accretion by black hole and out flowing wind (in case of super-Eddington) and added through fallback of the disrupted debris. We have simulated the light curve profiles in various spectral bands and fit them to the observations to determine the above mentioned physical parameters.
\end{abstract}

Keywords. Galaxy: nucleus, black hole physics, accretion, accretion disks

\section{Introduction}

A star is tidally disrupted if its pericenter $r_{p} \leqslant r_{t}$ where $r_{t} \sim R_{\star}\left(M_{\bullet} / M_{\star}\right)^{1 / 3}$ is the tidal radius and these events are called as tidal disruption events (TDEs) (Rees (1988)). Mageshwaran \& Mangalam (2015) have constructed a detail stellar dynamical model of TDE using parameters that include black hole $(\mathrm{BH})$ mass $M_{\bullet}$, specific orbital energy $E$ and angular momentum $J$, star mass $M_{\star}$ and radius $R_{\star}$. We define the dimensionless energy $\bar{e}=E / E_{m}$ and angular momentum $\ell=J / J_{l c}$ where $J_{l c}$ is the loss cone angular momentum and $E_{m}=G M_{\bullet} / r_{t}$ is the maximum orbital energy such that $J_{l c}=0$.

We have considered a self similar model of a time dependent and non-relativistic accretion disk for both sub-Eddington and super-Eddington disk with wind outflows due to strong radiative pressure. We have also considered a general viscosity prescription given by $\Pi_{r \phi}=-K \Sigma_{d}^{b} r^{d}$ where $\Sigma_{d}$ is the surface density of the disk, $r$ is the radius, $K, b$ and $d$ are constants (Mangalam (2001)). We assume a $\alpha$ viscous model for sub-Eddington disk with gas pressure dominating such that $b=5 / 3$ and $d=-1 / 2$ and the radiative viscosity for super-Eddington disk with radiation pressure given by $P_{r} \propto \rho^{\gamma}$ where $\gamma=4 / 3$ such that $b=1 / 3$ and $d=-5 / 6$ (Mangalam (2003)). The non relativistic disk equations in the limit of $u_{r} \ll u_{\phi}$, where $u_{r}$ is the radial velocity and $u_{\phi}$ is the azimuthal velocity, are given by $\dot{\Sigma}_{d}=-(1 / r) \partial_{r}\left(r u_{r} \Sigma_{d}\right)-\dot{\Sigma}_{w} ; \omega^{2}(r)=$ $(1 / r) \partial_{r} \Phi(r) ; u_{r} \Sigma_{d} \partial_{r}\left(r^{2} \omega\right)+\dot{\Sigma}_{w} r^{2} \omega(r)=-(1 / r) \partial_{r}\left(r^{2} \Pi_{r \phi}\right)$, where $\dot{\Sigma}_{d}$ and $\dot{\Sigma}_{w}$ are time derivative of the surface density of disk and wind components respectively and $\dot{\Sigma}_{w}$ is zero for sub-Eddington disk and $\Phi(r)=-G M_{\bullet} / r$. For a super-Eddington disk, assuming hydrostatic equilibrium up to the height $Z=Z_{p h}$ we construct the vertical density structure $\rho$ which is then used in the Eddington approximation to derive $Z_{p h}$. Using vertical momentum equation at $Z_{p h}$, implies $\dot{\Sigma}_{w} \propto \Sigma_{d}^{e} r^{f} \sqrt{1-\eta / \eta_{p h}}$, where $e=0.12$, $f=-3 / 2, \eta$ and $\eta_{p h}$ is the ratio of radiation pressure to total pressure at the mid plane and photosphere of the disk respectively. The self similar form of the accretion disk is 

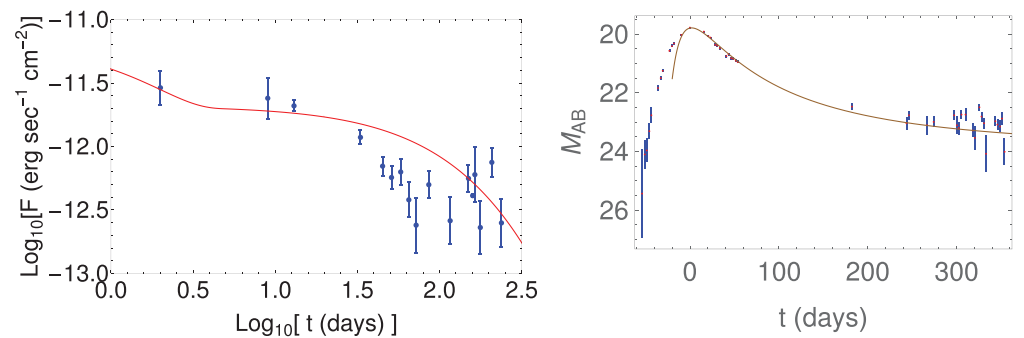

Figure 1. (Left) The sub-Eddington model fit to the XMMSL1 J061927.1-655311 (Saxton (2014)) in X-ray band. (Right) The super-Eddington model fit to the PS1-10jh (Gezari et al.(2012)) observations in optical g band.

taken to be $\Sigma_{d}=\Sigma_{0}\left(t / t_{0}\right)^{\beta} A \xi^{p}$ and $\xi=r / r_{0}\left(t / t_{0}\right)^{-\alpha}$, where $\Sigma_{0}, r_{0}, t_{0}, A, \alpha, \beta, p$ are the constants derived using disk equations and the mass of disk $M_{d}\left(t_{0}\right)=\int_{t_{m}}^{t_{0}} \dot{M}_{f b}(t) \mathrm{d} t$, where $\dot{M}_{f b}$ and $t_{m}$ are the infall rate of disrupted debris after disruption and orbital period of innermost debris respectively as given in Mageshwaran \& Mangalam (2015).

We assume a seed accretion disk formed by the circularization of the debris with initial mass $M_{d}\left(t_{0}\right)$ and the derived self similar structure of $\Sigma_{d}$ and $r_{0}=q r_{i n}$, where $r_{i n}$ is the inner radius taken to be innermost stable circular orbit $r_{I S C O}$ which is a function of black hole spin $j$. This seed disk evolves in effect of mass addition by the infall of disrupted debris and mass loss due to accretion by black hole and wind such that the structure of $\Sigma_{d}$ remains same. Applying mass conservation, $\dot{M}_{d}=\dot{M}_{f b}-\dot{M}_{a}-\dot{M}_{w}$ where $\dot{M}_{d}, \dot{M}_{a}$ and $\dot{M}_{w}$ are the rate of the change of disk mass, accretion rate by the black hole and mass loss due to out flowing wind respectively, we calculate the evolution of outer radius $r_{\text {out }}(t)$. The temperature of the sub-Eddington disk is then given by $\sigma T_{e}^{4}=(3 / 8) \omega \Pi_{r \phi}$ where $\omega=\sqrt{G M_{\bullet} / r^{3}}$ and for super-Eddington disk, the temperature is derived using radiative Eddington approximation at $Z_{p h}$. We now discuss the light curve profiles in various spectral bands fit to the observations and estimated physical parameters.

\section{Conclusions}

We have obtained the self similar structure of a time dependent and non relativistic accretion disk. The parameters $\{\beta, \alpha, p\}$ are $\{-8 / 7,5 / 21,3 / 2\}$ for sub-Eddington disk and $\{-0.95,1.22,-0.77\}$ for super-Eddington disk. The bolometric luminosity for sub-Eddington disk is $L \propto t^{-1.9}\left(\xi_{\text {out }}^{5 / 2}(t)-\xi_{\text {in }}^{5 / 2}(t)\right)$ and for super-Eddington disk is $L \propto t^{1 / 2}\left(\xi_{\text {out }}^{0.4}(t)-\xi_{\text {in }}^{0.4}(t)\right)$. The Fig 1 (Left) shows the sub-Eddington model fit to the observations in X-rays for XMMSL1 J061927.1-655311 and the derived parameters are $\bar{e}=0.00316, \ell=0.9, M_{6}=3.15, m=1.77, q=2$ and black hole spin $j=0.15$. The Fig 1 (right) shows the super-Eddington model fit to the PS1-10jh observations in optical $\mathrm{g}$ band and the derived parameters are $\bar{e}=0.0001, \ell=1, M_{6}=36, m=1.56, q=2, j=$ 0.5 .

\section{References}

Gezari et al. 2012, Nature, 485, 217

Mageshwaran, T. \& Mangalam, A. 2015, ApJ, 814, 141

Mangalam, A. 2001, A\&A A, 379, 1138

Mangalam, A. 2003, Bulletin of the Astronomical Society of India, 31, 207

Rees, Martin J. 1988, Nature, 333, 523

Saxton et al. 2014, A\& A, 572

Strubbe, Linda E. \& Quataert, Eliot. 2009, MNRAS, 400, 2070 